\title{
Evaluation of the collagen-boosting effects of a Moldavian dragonhead extract.
}

Franziska Wandrey $\mathbf{P h D}^{1}$, Christina Pickel $\mathbf{P h D}^{1}$, Elisabeth Jongsma ${ }^{2}$, Prof. Collin Y. Ewald $\mathbf{P h D}^{2}$, Torsten Grothe $\mathrm{PhD}^{1 *}$

${ }^{1}$ Mibelle Group Biochemistry, Mibelle AG, Bolimattstrasse 1, 5033 Buchs AG, Switzerland

${ }^{2}$ Extracellular Matrix Regeneration Laboratory, Institute of Translational Medicine, Department of Health Sciences and Technology, Eidgenössische Technische Hochschule (ETH) Zürich, Schorenstrasse 16, 8603 Schwerzenbach ZH, Switzerland

*Corresponding Author: Torsten Grothe, Mibelle Group Biochemistry, Mibelle AG, Bolimattstrasse 1, 5033 Buchs AG, Switzerland.

Received date: 23 July 2021; Accepted date: 02 August 2021; Published date: 06 August 2021

Citation: Wandrey F, Pickel C, Jongsma E, Ewald CY, Grothe T (2021) Evaluation of the collagen-boosting effects of a Moldavian dragonhead extract. J Comm Med and Pub Health Rep 2(7): https://doi.org/10.38207/jcmphr20210102

Copyright: (C) 2021 Torsten Grothe. This is an open-access article distributed under the terms of the Creative Commons Attribution License, which permits unrestricted use, distribution, and reproduction in any medium, provided the original author and source are credited.

\begin{abstract}
Skin aging is a natural process that is influenced by various intrinsic and extrinsic factors such as UV radiation, pollution, oxidative stress, or an unhealthy lifestyle. Premature skin aging affects millions of people worldwide, but treatment options are limited and often of invasive nature. Therefore, the demand for alternative natural and safe products for nutraceutical use is increasing. Moldavian dragonhead is known for its high content in flavonoid glucuronides and its antioxidative effects. However, its effect on skin appearance parameters is unknown to date. Our in-vitro study showed that treatment of mouse C2C12 cells with Moldavian dragonhead extract activates the innate longevity pathway involving the signaling kinase AMPK and the transcription factor FOXO1. In vivo, Moldavian dragonhead extract had a collagen-boosting effect preserving a youthful collagen expression and mass during aging in Caenorhabditis elegans. Moreover, in humans, daily food supplementation with $200 \mathrm{mg}$ Moldavian dragonhead dry extract (DracoBelle ${ }^{\mathrm{TM}} \mathrm{Nu}$ ) for eight weeks in an open pilot trial significantly increased skin moisturization and elasticity by $14.4 \%$ and $6.7 \%$, respectively. Furthermore, skin density was increased as shown by ultrasound visualization. There were no reports of adverse events during the study period. We, therefore, conclude that Moldavian dragonhead extract presents a safe and effective treatment option for (photo) aged skin
\end{abstract}

Keywords: Moldavian dragonhead, collagen-boosting, anti-aging, AMPK, FOXO, C. elegans, flavonoid glucuronides

\section{Introduction}

A flawless and young appearance of skin and hair is an important factor reflecting general well-being and, although being a natural process, aging and obvious signs thereof are perceived as unhealthy. Multiple intrinsic and extrinsic factors affect skin health, leading to structural, metabolic, and physiological changes and ultimately visible signs of premature aging [41]. This is characterized by an increase in oxidative stress caused by reactive oxygen species (ROS), a reduction of skin cell proliferation and thus skin renewal as well as an increased degradation of the skin matrix, which is composed of collagen and elastin fibers [41]. The damage of connective tissues, such as fragmentation, oxidation, and glycation of collagen, accumulates during aging [4]. These changes at the molecular level affect tissue integrity and lead to a continuous decline in collagen mass, which ultimately results in the formation of wrinkles and sagging skin.

Anti-aging strategies have been targeting these processes to prevent or reverse premature skin aging. However, besides topical application of daily skincare products or pharmacological agents, many currently available skin anti-aging treatments involve invasive procedures such as chemical peelings or injection of hyaluronic acid or botulinum neurotoxin [8]. Implementing the rather novel concept of "beauty from within", systemic treatment has been established for the prevention of visible skin aging in the past years, mostly involving hormone replacement or antioxidant therapy [8]. But these treatments often harbor the risk of diverse side effects, which causes dissatisfaction in patients. Natural ingredients provide a potential solution to these concerns and offer a safe, non-invasive alternative for the prevention or improvement of premature skin aging.

Moldavian dragonhead [3] is a perennial plant native to Central Asia and has been introduced and naturalized to Eurasia and Northern America. Being a member of the mint family and having a pleasant lemon balm-like odor and taste, the aerial parts of Moldavian dragonhead have gained popularity for the preparation of culinary tea or flavoring of dishes. Traditionally, it has been used as a herbal drug to treat headaches, stomach and liver disorders, or congestions [24] and it has additional cardioprotective effects $[3,10,13,17,19,32]$. Moreover, the antioxidant activity of D. moldavica has been studied by multiple researchers in vitro $[3,22,23]$. Extracts of D. moldavica 
are rich in secondary metabolites such as flavonoids and flavonoid glucuronides, and tilianin, luteolin-7-glucuronide, and apigenin-7glucuronide are discussed as the major actives of the herb $[\mathbf{6}, \mathbf{1 1}, \mathbf{3 5}$, 36, 37, 38]. These secondary metabolites have been discussed to protect cells from oxidative stress and apoptosis, reduce inflammations, and have cardio- and neuroprotective activities $[\mathbf{1 , 1 8}$, 42]. Interestingly, there is no information available to date about the effect of oral consumption of an extract of Moldavian dragonhead on skin parameters and signs of premature aging. Therefore, this study aimed at determining the effect of Moldavian dragonhead extract on the 5' adenosine monophosphate (AMP)- activated protein kinase (AMPK)- fork head box protein O1 (FOXO1) signaling pathway which has been linked to stem cell longevity and aging [27]. Previous studies showed that FOXO1 is activated upon caloric restriction, a dietary regimen that involves reduced calorie intake while maintaining optimal levels of protein, vitamins, and minerals [16]. The beneficial effect of caloric restriction on skin cells was shown upon topical treatment with an extract of snow algae (Coenochloris signiensis) which mimics caloric restriction by increasing the expression of the anti-aging protein hormone Klotho and activating

\section{Materials and methods}

Preparation and analysis of an extract from Dracocephalum moldavica $\mathbf{L}$.

$3 \%$ Dracocephalum moldavica L. aerial parts were extracted with 30 $\%$ ethanol (w/w) by percolation. Following the first extraction of 3 hours at $50^{\circ} \mathrm{C}$, the supernatant was stored and replaced by fresh solvent. Following further extraction for 3 hours at $50^{\circ} \mathrm{C}$, the supernatants were pooled, leading to a final extracted dry mass of 1.5 $\%$. The extract was analyzed using a Sun Fire C18 column $(3.5 \mu \mathrm{m}$; 3.0 x $50 \mathrm{~mm}$; Waters AG, Baden, Switzerland) connected to a highperformance liquid chromatography (HPLC) system with UV, electrospray ionization mass spectrometry, and evaporative light

\section{Protein analysis by immunoblot}

C2C12 murine myoblast cells were treated with $0.5 \%$ Moldavian dragonhead extract for 10 minutes, washed with ice-cold phosphate buffer saline (PBS), and lysed in respective buffer (42 mM Tris-HCl, $1.3 \%$ sodium dodecyl sulphate (SDS), $6.5 \%$ glycerine, $100 \mu \mathrm{M}$ sodium orthovanadate containing $2 \%$ phosphatase and protease inhibitors). Protein concentration was determined using the bicinchoninic acid (BCA) assay and equal amounts of protein were separated by SDS polyacrylamide gel electrophoreses. Following transfer to polyvinylidene fluoride (PVDF) membranes and blocking

\section{Determination of hydroxyproline content in Caenorhabditis} elegans

A stock solution of Moldavian dragonhead extract was prepared by dissolving the powder extract in water and subsequent sterile
AMPK. This leads to an increase in the expression of collagens and a decrease in the expression of matrix metalloproteinases (MMPs). The rejuvenating effect of a snow algae extract has been confirmed in clinical studies, in which it smoothened the cutaneous microrelief of Crow's feet wrinkles and led to an increase in the collagen/elastin ratio [26]. Interestingly, increased FOXO1 signaling was shown to increase the expression of type 1 collagen (COL1A 1 ), the most abundant collagen in humans which is also present in the dermis of the skin [29]. On the other hand, reduced FOXO1 expression, for example, induced by ultraviolet (UV) irradiation, decreases COL1A1 expression and increases expression of MMPs which degrade collagen [29]. This suggests a direct link between FOXO1 signaling and collagen levels as well as skin aging. Therefore, the effect of Moldavian dragonhead extract on collagen levels was further analyzed in the nematode Caenorhabditis elegans (C. elegans), an established model organism in aging research. Furthermore, the third objective of this research program was an assessment of the anti-aging efficacy of Moldavian dragonhead extract upon daily consumption as a food supplement in a panel of volunteers with photoaged skin.

scattering (ELS) detectors (Shimadzu, Kyoto, Japan). The run was performed using solvent $\mathrm{A}\left(\mathrm{H}_{2} \mathrm{O}+0.1 \%\right.$ formic acid $)$ and solvent $\mathrm{B}$ $(\mathrm{MeCN}+0.1 \%$ formic acid) with a gradient of 5-100\% solvent $\mathrm{B}$ over 30 minutes followed by a wash-out with $100 \%$ solvent B for 10 minutes. Scan ranges were set to $190-600 \mathrm{~nm}$ for UV and +/switching 160-1500 mass/charge (m/z). Identification of compounds in the extract was based on UV, mass, and elution order as previously described [39]. The subsequent studies were performed using the described liquid extract or a powder version prepared by spraygranulation of the liquid extract on an isomalt carrier (DracoBelle ${ }^{\mathrm{TM}}$ $\mathrm{Nu}$, provided by Mibelle Group Biochemistry, Buchs, Switzerland).

with $5 \%$ milk solution in Tris-buffered saline (TBS) containing $0.1 \%$ Tween-20, proteins were visualized with antibodies against phosphorylated AMPK $\alpha$ (Cell Signaling Technology, Danvers, MA, USA), total AMPK $\alpha$ (Cell Signaling Technology), phosphorylated FOXO1 (Thr24; Cell Signaling Technology) and $\beta$-Actin (SigmaAldrich, St. Louis, MI, USA). Protein amounts were quantified by densitometric analysis using ImageJ software (NIH, USA) and phosphorylated protein was normalized to total protein or loading control. The experiment was performed in triplicate.

filtration. The Moldavian dragonhead extract was mixed in the food of C. elegans (OP-50) and seeded onto Nematode growth medium (NGM) culturing plates at final concentrations of $0.1 \mathrm{mg} / \mathrm{ml}$ and 1 $\mathrm{mg} / \mathrm{ml}$, respectively. The determination of the amount of 
hydroxyproline (Hyp) was performed as previously described [31]. Briefly, synchronized wild-type background with temperaturesensitive sterile mutations (TJ1060 spe-9(hc88) I; rrf-3(b26) II.) $C$. elegans at larval stage 1 were cultured on NGM plates, foodsupplemented or not with Moldavian dragonhead extract at $25^{\circ} \mathrm{C}$. On day 5 of adulthood, the aged $C$. elegans were hydrolysed using hydrochloric acid and the amount of hydroxyproline, as well as the total protein amount, was determined [31]. The hydroxyproline assay and protein assay was performed using the QuickZyme Total Collagen and Total Protein Kits (QuickZyme Biosciences, Leiden,

\section{In-vivo assessing collagen expression in $C$. elegans}

Collagen expression was quantified in the $C$. elegans strain LSD2002 (spe-9(hc88) I; xchIs001 [Pcol-144: GFP; pha-1(+)] X) expressing a green fluorescent protein (GFP) under the control of the col-144 collagen promoter. The amount of green fluorescence in these transgenic animals reflects gene expression levels of collagen [5] Synchronized C. elegans at larval stage 1 were cultured on NGM plates, food-supplemented with $0.0 \mathrm{mg} / \mathrm{mL}, 0.1 \mathrm{mg} / \mathrm{ml}$ or $1 \mathrm{mg} / \mathrm{ml}$ Moldavian dragonhead extract at $25^{\circ} \mathrm{C}$. On day 5 of adulthood, images were taken with an upright brightfield fluorescence

\section{Food supplement study}

32 female Caucasian volunteers (41 to 64 years), phototype I-III, with sun-damaged skin were included in a pilot study in Switzerland to test the anti-aging effect of Moldavian dragonhead extract (DracoBelle ${ }^{\mathrm{TM}}$ $\mathrm{Nu}$ ) on the skin. Subjects with an ethnicity other than Caucasian, a phototype > III, a severe systemic or dermatologic disease, using any topical (drug-containing) or cosmetic anti-aging product on the test area within the previous four weeks, taking any kind of dietary supplement within the previous eight weeks, or participating in any other clinical study within the previous eight weeks were excluded from this study.

During a pre-conditioning phase of 14 days before the study start, subjects refrained from using anti-aging products on the inner forearm. Following baseline determination (t0) of skin hydration

\section{Statistical analysis}

For all measured parameters, paired data were tested by nonparametric permutation analysis using the StatXact software (version 5.0.1.; Cytel Ltd., USA). A p-value of less than 0.05 was considered

\section{Results}

\section{Moldavian dragonhead extract contains flavonoid glucuronides}

To assess the phytochemical constitution of the extract prepared from the aerial parts of Moldavian dragonhead, it was analyzed by chromatography followed by combined UV, mass spectrometry, and ELS detection. Based on the obtained UV spectra, the mass and
Netherlands) according to the manufacturer's instructions. The collagen assay uses a colorimetric determination of hydroxyproline residues that is based on the oxidation of Hyp to a pyrrole intermediate, followed by reaction with Ehrlich's reagent dissolved in concentrated perchloric acid $\left(\mathrm{HCIO}_{4}\right)$ [31]. The optical density of the sample at $560 \mathrm{~nm}$ was compared to a collagen standard for the quantitative determination of collagen content [31]. The determination of hydroxyproline and total protein amount was performed in duplicates and two independent assays.

microscope (200x magnification) and GFP intensity was measured using ImageJ software, and Rstudio was used for data processing. Autofluorescence in the GFP filter range was blocked using an established GFP filter setup [30]. The expression of col-144 was scored by imaging and analysis of GFP intensity and the col-144 expression was calculated as GFP intensity per surface area of the animal. For each condition, >20 C. elegans were imaged and analyzed.

(Corneometer® CM 825 MPA, Courage \& Khazaka electronic $\mathrm{GmbH}$, Cologne, Germany), skin elasticity (Cutometer® dual MPA 580 , Courage \& Khazaka electronic $\mathrm{GmbH}$ ) and epidermis as well as dermis density (DermaScan ${ }^{\circledR}$ C, Cortex Technology, Hadsund, Denmark) on the inner forearm, volunteers consumed $200 \mathrm{mg}$ DracoBelle $^{\mathrm{TM}} \mathrm{Nu}$ dissolved in 200-250 $\mathrm{ml}$ of cold liquid of their choices such as water or fruit juice once daily for 2 months. After 56 days ( $\mathrm{t} 1)$ of intake of DracoBelle ${ }^{\mathrm{TM}} \mathrm{Nu}$, the test parameters were measured again, and a self-assessment questionnaire was filled out. The study was performed at the Skin Test Institute, Neuchâtel, Switzerland in accordance with the ethical principles for medical research in human subjects (Declaration of Helsinki). All participants gave informed consent after study details were explained.

statistically significant. Unless stated differently, data are depicted as the mean plus standard error of the mean.

elution order, the main flavonoid glucuronides were identified in the extract and matched to a previous description [39] (Figure 1A). The obtained peaks 1-6 could therefore potentially be assigned to luteolinglucuronide, apigenin-glucuronide, tilianin, diosmetin-glucuronide, 
acacetin-glucuronide, and an unknown compound with a flavonoidtype UV spectrum, respectively (Figure 1B). The here observed changes in retention times of compounds likely differ from literature

A

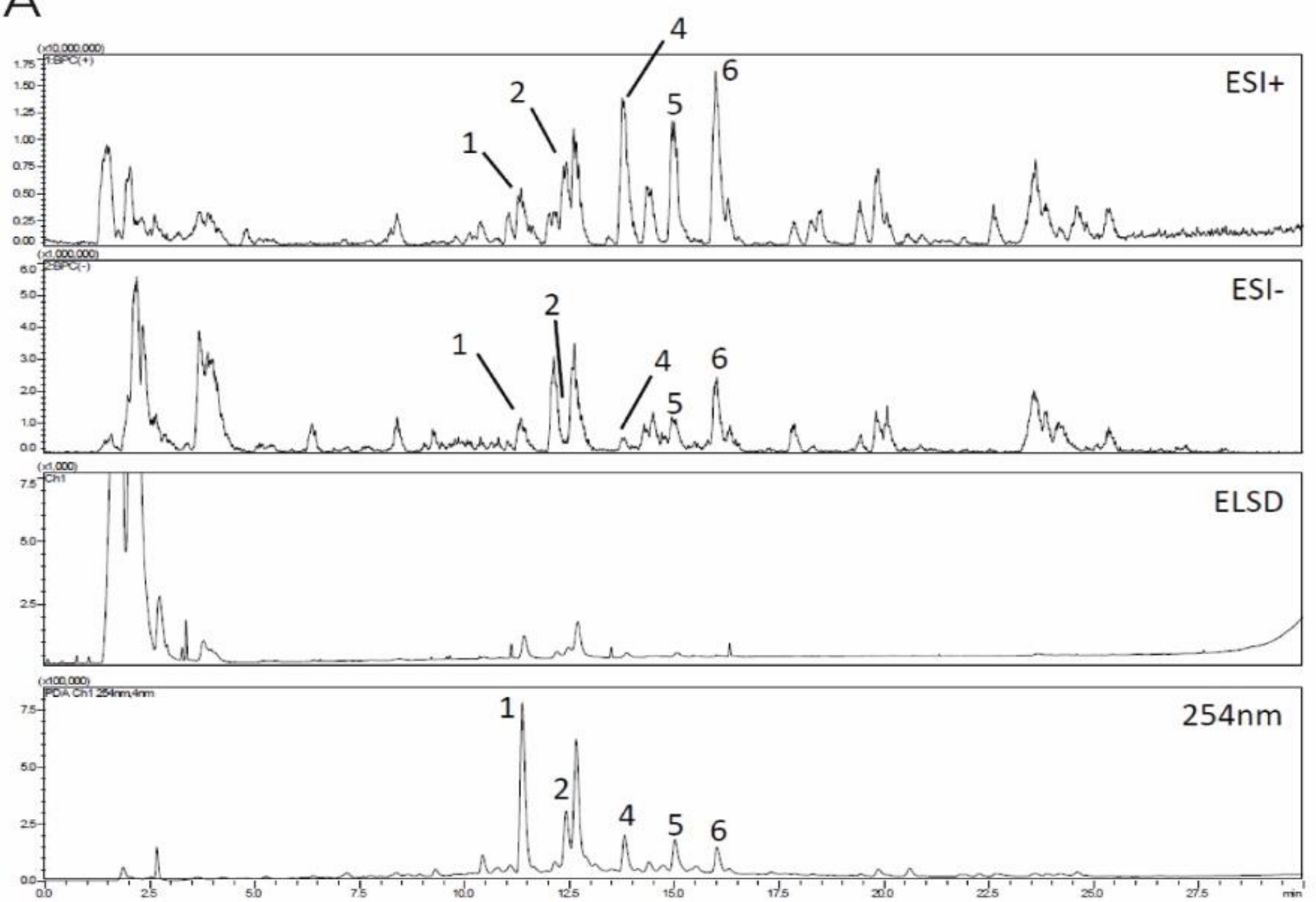

B

Peak 1: Luteolin-glucuronide<smiles>O=C(O)C1OC(Oc2cc(O)c3c(=O)cc(-c4ccc(O)c(O)c4)oc3c2)C(O)C(O)C1O</smiles>

Peak 4: Talianin<smiles></smiles>

Peak 5b: Acacetin-glucuronide<smiles>COc1ccc(-c2cc(=O)c3c(O)cc(OC4OC(C(=O)O)C(O)C(O)C4O)cc3o2)cc1</smiles>

due to different analytical conditions such as stationary phase and gradient. 


\section{Moldavian dragonhead extract activates the AMPK/FOXO1} signalling pathway

Treatment of $\mathrm{C} 2 \mathrm{C} 12$ cells with $0.5 \%$ Moldavian dragonhead extract for $10 \mathrm{~min}$ led to a 3.96-fold increase in AMPK phosphorylation compared to treatment with solvent only (Figure 2). FOXO1 phosphorylation was reduced 2.63-fold upon treatment with $0.5 \%$
Moldavian dragonhead extract, leading to a respective increase in active, non-phosphorylated protein (Figure 2). Together, these results indicate that Moldavian dragonhead extract activates the AMPKFOXO1 signaling pathway, a key pathway regulating various antiaging mechanisms and boosting collagen expression.

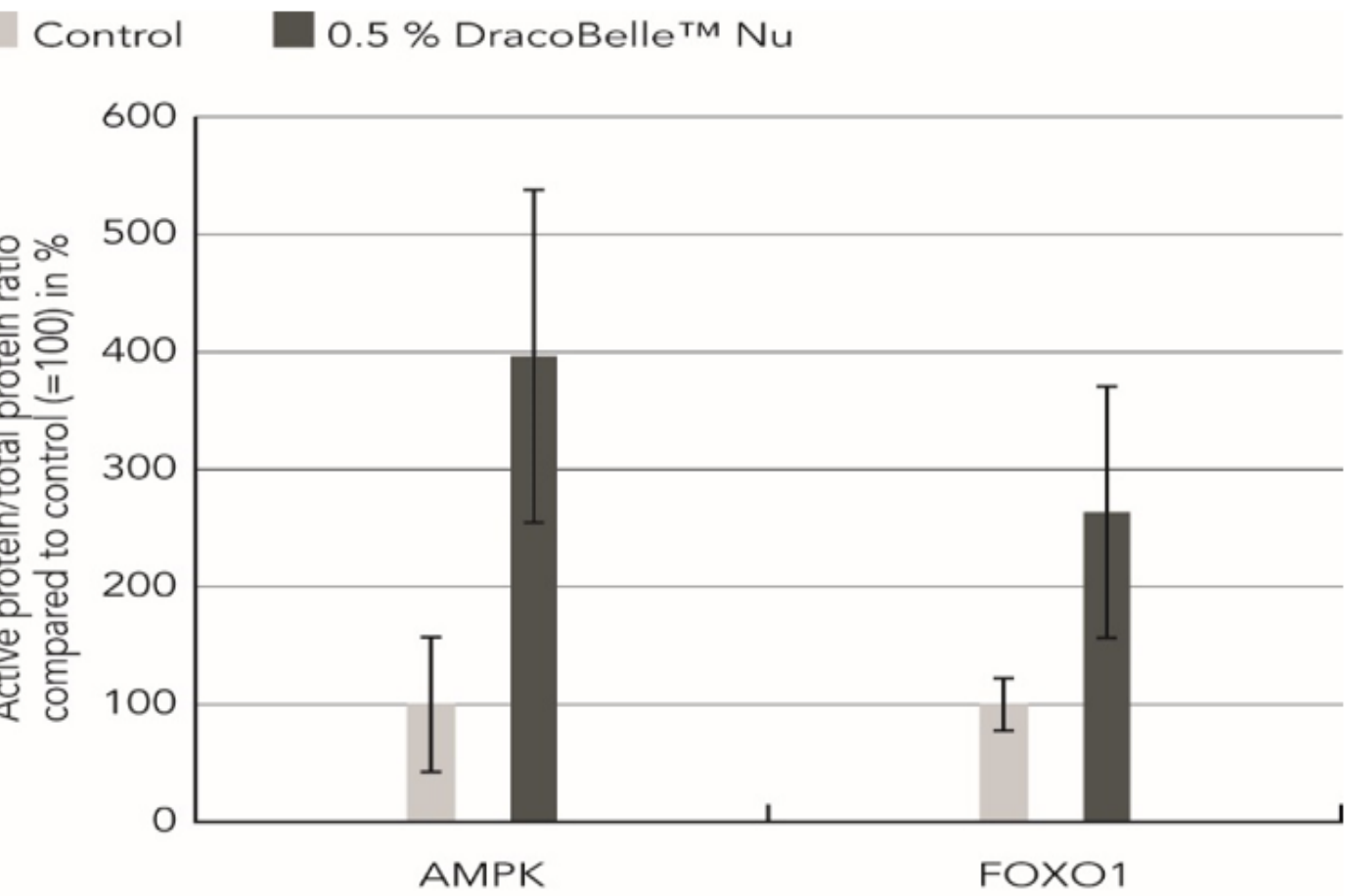

Figure 2: Effect of Moldavian dragonhead extract on AMPK and FOXO1 activation. C2C12 cells were treated with $0.5 \%$ Moldavian dragonhead extract for 10 minutes followed by an analysis of AMPK, P-AMPK, P-FOXO1, and $\beta$-Actin protein levels by immunoblot.

\section{Moldavian dragonhead extract boosts the expression of collagen} during aging in Caenorhabditis elegans

During aging, collagen biosynthesis and collagen mass continuously decline across species [4] Longevity interventions, such as activation of the AMPK-FOXO1 signaling pathway, preserve and prevent this age-dependent collagen decline in model organisms such as $C$. elegans [5]. To determine whether the Moldavian dragonhead extract could also act as a longevity-promoting intervention to preserve youthful collagen levels, we applied Moldavian dragonhead extract to the $C$. elegans food. The total collagen levels were assessed by the determination of hydroxyproline ((2S,4R)-4-hydroxyproline; Hyp) a modified amino acid almost exclusively found in the triple helix repeats of the collagen protein. Its amount in tissue hydrolysates is, therefore, a direct measure of the amount of collagen present. On day 5 of adulthood at $25^{\circ} \mathrm{C}$, a time-point marked age-related loss of collagen mass [5], the collagen levels in this aged C. elegans treated with Moldavian dragonhead extract were significantly higher than in untreated C. elegans ( $<$ 0.05) (Figure 3A), clearly demonstrating a collagen-boosting effect. This effect of Moldavian dragonhead extract was concentration-dependent, leading to an increase in the collagen abundance by $49.8 \%$ at $0.1 \mathrm{mg} / \mathrm{ml}$ Moldavian dragonhead extract and an increase of $65.9 \%$ upon treatment with $1 \mathrm{mg} / \mathrm{ml}$ Moldavian dragonhead extract (Figure 3A).

To confirm these results, a second study was performed using the $C$. elegans strain LSD2002 in which the expression of the fluorescent protein GFP is coupled to the activation of the col-144 collagen promoter, thereby directly linking the expression of collagen with an increase in fluorescent signal. In comparison to untreated C. elegans, aged transgenic animals treated with 0.1 and $1 \mathrm{mg} / \mathrm{ml}$ Moldavian dragonhead extract showed an increased expression of the col-144 promoter at day- 5 of adulthood at $25^{\circ} \mathrm{C}$. This was visible in images taken of aged transgenic animals following the treatment (Figure 3B) as well as quantifications of the GFP fluorescence (Figure 3C). The GFP fluorescence increased by $7.3 \%$ upon treatment with $0.1 \mathrm{mg} / \mathrm{ml}$ Moldavian dragonhead extract, and by $28.6 \%$ upon treatment with 1 $\mathrm{mg} / \mathrm{ml}$ Moldavian dragonhead extract, which was significant compared to untreated control (Figure 3C). Taken together, Moldavian dragonhead promotes collagen expression and preserves youthful collagen levels during aging. 


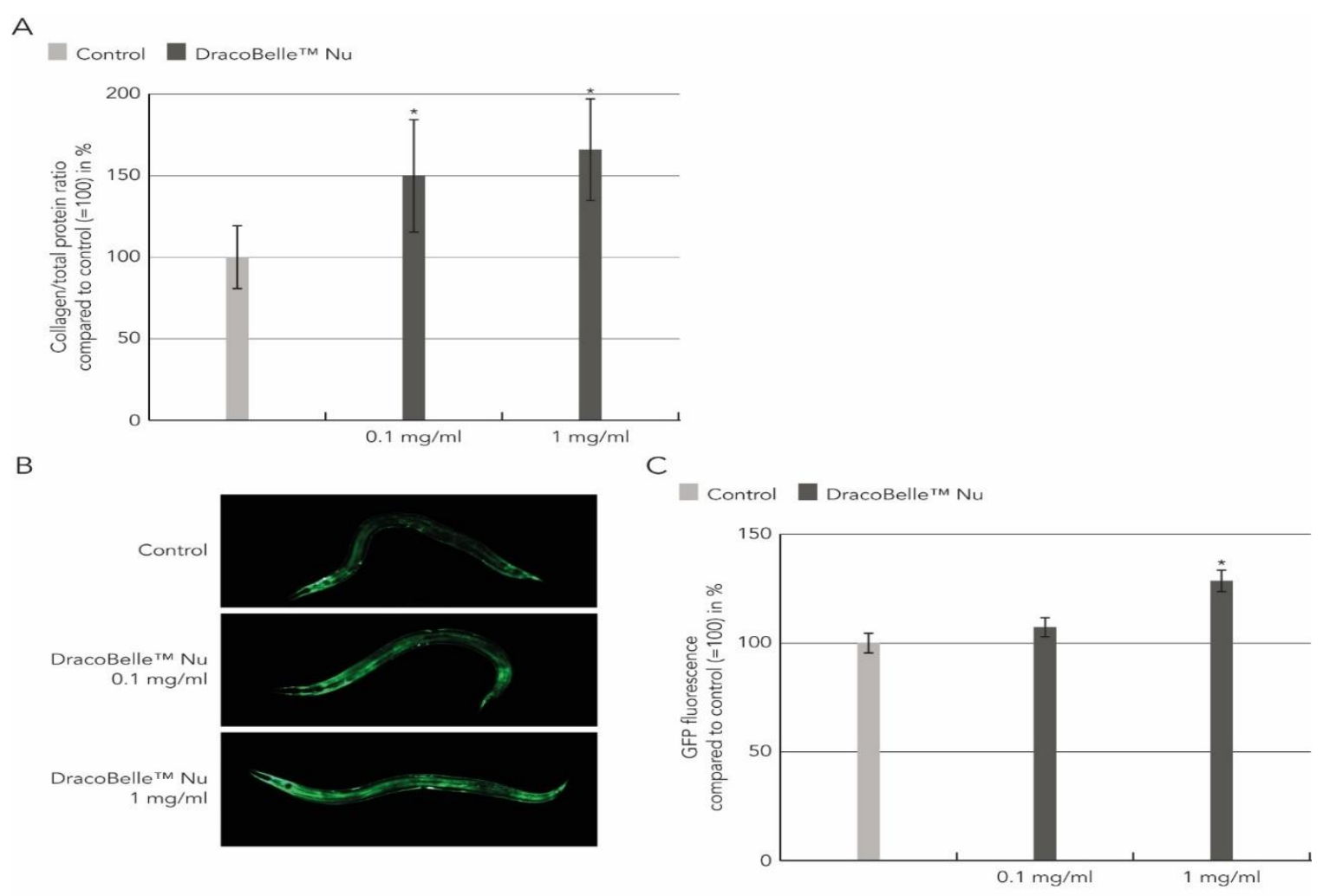

Figure 3: Collagen-boosting effect of Moldavian dragonhead in C. elegans.

(A) Wild-type C. elegans were treated with $0.1 \mathrm{mg} / \mathrm{ml}$ or $1 \mathrm{mg} / \mathrm{ml}$ Moldavian dragonhead extract for 7 days before analysis of the hydroxyproline and total protein levels at day- 5 of adulthood.

(B) The C. elegans strain LSD2002 expressing GFP under the control of the col-144 promoter was treated with $0.1 \mathrm{mg} / \mathrm{ml}$ or $1 \mathrm{mg} / \mathrm{ml} \mathrm{Moldavian}$ dragonhead extract for 7 days and the GFP fluorescence was assessed by fluorescence microscopy at day- 5 of adulthood.

(C) The GFP fluorescence was quantified using ImageJ software. * ${ }^{*}<0.05$.

Daily ingestion of DracoBelle ${ }^{\mathrm{TM}} \mathrm{Nu}$ leads to increased skin elasticity and density

To test the anti-aging and collagen-boosting effect of DracoBelle ${ }^{\mathrm{TM}}$ $\mathrm{Nu}$ in vivo, 32 subjects with sun-damaged skin were instructed to consume $200 \mathrm{mg}$ of the Moldavian dragonhead extract as a food supplement daily for 2 months. Compared to initial conditions, skin moisturization increased significantly by $14.4 \%$ from $29.2 \pm 5.5$ arbitrary units (AU) at baseline to $33.4 \pm 7.1 \mathrm{AU}$ at $\mathrm{t} 1(\mathrm{p}=0.0008$; Figure 4A). Skin elasticity increased by $6.7 \%$ from $0.771 \pm 0.178$ $\mathrm{AU}$ at t0 to $0.823 \pm 0.156 \mathrm{AU}$ after two months $(\mathrm{p}=0.0022$; Figure 4A). Moreover, epidermis and dermis density increased slightly by $2.5 \%$ compared to initial conditions. The increase in skin density was also visible on ultrasound pictures taken with a DermaScan ${ }^{\circledR} \mathrm{C}$,

\section{Discussion}

In this study, an extract prepared from the aerial parts of Dracocephalum moldavica $L$. was characterized for its phytochemical constitution and its effect on cellular signaling through the AMPK-FOXO1 axis as well as collagen-boosting and anti-aging efficacy. In line with previous descriptions, the hydro-ethanolic extract contained various flavonoid and flavonoid glucuronides such as apigenin-glucuronide and tilianin $[37,38,39]$ (Figure 1). In contrast, rosmarinic acid was not detected upon extraction of Moldavian dragonhead with $30 \%$ ethanol. Thus, there is an overall high similarity between the here prepared extract and the traditional Chinese medicine Yixin Badiranjibuya Granule, which is well-known and often used for the treatment of cardiovascular diseases [21, 39]. where a reduction of the number of low echogenic pixels was observed (Figure 4B). This represents an increase in extracellular matrix components such as collagen and elastin fibers, and a reduction of interstitial water content and consequently corresponds to an increase in dermal and epidermal density.

Subjects confirmed the beneficial and hydrating effect of a food supplementation with Moldavian dragonhead extract by a selfassessment. $94 \%$ of the volunteers reported that they feel better in their skin, while $72 \%$ confirmed that their skin feels more hydrated. Both results are statistically significant.

The food supplement was well tolerated by volunteers during the study, and no intolerance reactions were observed.

Analysis of the effect of Moldavian dragonhead extract on cellular signaling showed that it modulates the AMPK-FOXO1 signaling axis by increasing phosphorylation of AMPK and reducing phosphorylation of FOXO1, thereby activating the pathway (Figure 2). The role of this pathway in prolonging the lifespan and mediating longevity has been demonstrated in various species in the past years in the context of caloric restriction (CR) $[14,15,25,33,34]$. Interestingly, recent research showed that $\mathrm{CR}$ promotes metabolic changes and structural changes in the skin [7]. The observed increase in epidermal thickness in this study is described to be reminiscent of young tissue [7]. Moreover, and very interestingly, aging-related genes and pathways, including collagen remodelling, DNA damage 
repair, and cell metabolism, are regulated by FOXO transcription factors [33]. We, therefore, conclude that Moldavian dragonhead extract switches on a cellular anti-aging program involving AMPKFOXO1 signaling. In line with this, the results of this study show that the Moldavian dragonhead extract promotes the expression of collagen in the nematode $C$. elegans, a well-described model organism for aging research [5]. The Moldavian dragonhead extract increases both the activity of the collagen promoter, as shown using the collagen col-144 GFP reporter system, as well as the amount of collagen protein present in the organism, which was shown by quantification of hydroxyproline (Figure 3). Hyp is a modified amino acid formed from the amino acid proline by the Vitamin C-dependent enzyme prolyl-4-hydroxylase. The essential involvement of Vitamin $\mathrm{C}$ in this process is the reason for the European Food Safety Authority (EFSA)- approved collagen-related health claim: Vitamin C contributes to natural collagen formation for the normal function of the skin. Delaying the progressive decrease in the extracellular matrix by actively prolonging the biosynthesis of collagen in C. elegans was further shown to prolong the lifespan of the nematode $[4, \mathbf{5}, \mathbf{3 1}]$. Therefore, agents promoting extracellular matrix youthfulness may have a systemic effect in addition to reducing apparent signs of aging [5].

In order to translate the findings of these in-vitro and in-vivo studies to systemic effects in humans, a clinical study was performed with the aim to assess the anti-aging effect of Moldavian dragonhead extract. Besides demonstrating no adverse effects upon a daily supplementation of $200 \mathrm{mg}$ Moldavian dragonhead extract, this pilot study showed that daily intake of DracoBelle ${ }^{\mathrm{TM}} \mathrm{Nu}$ for two months leads to a significant increase in skin moisturization and elasticity (Figure 4A). Dry skin is one of the characteristics of photoaged skin [12], which can be improved by daily supplementation with Moldavian dragonhead extract. Aged and sun-damaged skin is moreover characterized by a decrease in elastic fibers and extracellular matrix, which is concurrent with a loss of skin elasticity [28]. Dermis and epidermis density increased visibly, and the subepidermal low echogenicity band (SLEB) was reduced after 56 days of treatment with Moldavian dragonhead extract (Figure 4B), reflecting an increase in well-arranged extracellular matrix proteins and a decrease in unbound water content [9]. This is in line with an increase in skin elasticity following the treatment with Moldavian dragonhead extract (Figure 4A). These results match a previous description of the beneficial effect of topical flavonoid treatment on aged skin [2]. Moreover, flavonoid glucuronides, which are represented in the Moldavian dragonhead extract at significant levels, have been shown to have protective effects concerning UVA- and UVB-induced photo-aging [20, 43].

In conclusion, food supplements containing Moldavian dragonhead extract may ameliorate the appearance and feel of sun-damaged or aged skin by improving skin hydration and elastic fiber distribution without side effects. This is potentially regulated through activation of the innate longevity pathway involving AMPK and FOXO1 and an increase in the amount of collagen present in the skin. Confirmation of the effectiveness through placebo-controlled interventions is desirable.
A

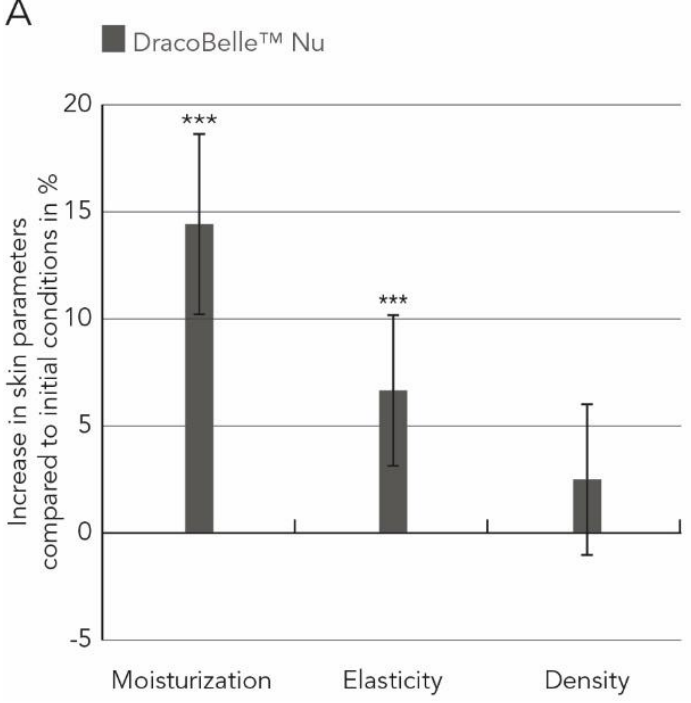

B

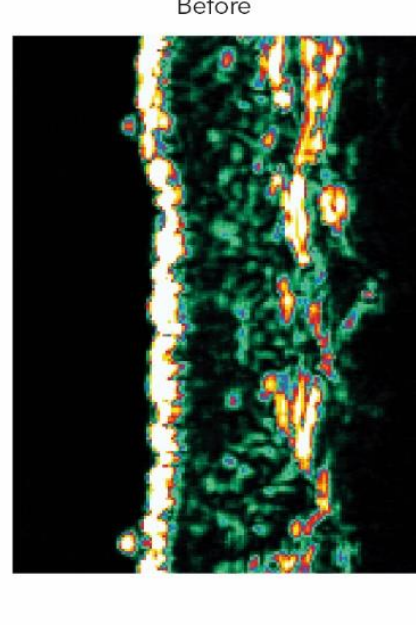

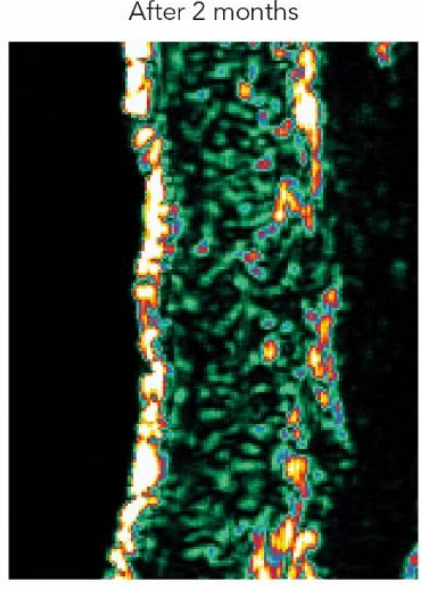

Figure 4: Changes in skin moisturization, elasticity, and density following oral supplementation treatment with Moldavian dragonhead extract. (A) Skin parameters were determined at baseline and after two months of ingestion of a Moldavian dragonhead extract and changes (in \%) compared to baseline are depicted. $* * * p<0.005$.

(B) Visualization of dermis and epidermis density by ultrasound before and after two months of treatment with Moldavian dragonhead extract. Bright areas represent tissue with high density, reflecting the ultrasound waves, whereas black regions are regions of low dermal density.

\section{Acknowledgments}

This study was sponsored and funded by a grant from the Mibelle Group Biochemistry, Mibelle AG, Switzerland. We acknowledge our colleagues Daniel Schmid and Cornelia Schuerch for scientific support and Riikka Sacher for sample preparation and Prof. Matthias Hamburger, University of Basel, for the phytochemical characterization of the extract. 


\section{Abbreviations}

AMPK 5' adenosine monophosphate (AMP)-activated protein kinase, AU arbitrary units, BCA bicinchoninic acid, CR caloric restriction, DNA deoxyribonucleic acid, ECM extracellular matrix, EFSA European Food Safety Authority, ELS evaporative light scattering, ESI electrospray ionization, FOXO fork head box protein O1, HPLC high-performance liquid chromatography, Hyp hydroxyproline, MMP matrix metalloproteinase, MS mass

\section{References}

1. Akanda R, Uddin N, Kim I.-S, Ahn D, Tae HJ, et al. (2019) The biological and pharmacological roles of polyphenol flavonoid tilianin. Eur. J. Pharmacol. 842: 291-297.

2. Crisan D, Crisan M, Moldovan M, Lupsor M, Badea R (2012) Ultrasonographic assessment of the cutaneous changes induced by topical flavonoids. Clin. Cosmet. and Investig. Dermatol. 5: 7 13.

3. Dastmalchi K, Dorman HJD, Kosar M, Hiltunen R (2007) Chemical composition and in vitro antioxidant evaluation of a water-soluble Moldavian balm (Dracocephalum moldavica L.) extract. Food. Sci. Technol. 40(2): 239-248.

4. Ewald CY (2020) The Matrisome during Aging and Longevity: A Systems-Level Approach towards Defining Matreotypes Promoting Healthy Aging. Gerontology 66(3): 266-274.

5. Ewald CY, Landis JN, Abate JP, Murphy CT, T.K. Blackwell TK (2015) Dauer-independent insulin/IGF-1-signalling Implicates Collagen Remodelling in Longevity. Nature 519(7541): 97-101.

6. Feng, C.-G. and Q. Li. Review of chemical constituents of Dracocephalum moldavica L. and their pharmacological activities. Chinese Traditional Patent Medicine 25(2): 154-156, 2003.

7. Forni MF, Peloggia J, Braga TT, Ortega Chinchilla JE, Shinohara J, et al. (2017) Caloric Restriction Promotes Structural and Metabolic Changes in the Skin. Cell Rep. 20(11): 2678-2692.

8. Ganceviciene R, Laikou AI, Theodoridis A, Makrantonaki E, Zouboulis CC (2012) Skin anti-aging strategies. Dermatoendocrinol. 4(3): 308-319.

9. Gniadecka M, Gniadecki R, Serup J, Sondergaard J (1994) Ultrasound Structure and Digital Image Analysis of the Subepidermal Low Echogenic Band in Aged Human Skin: Diurnal Changes and Interindividual Variability. J. Invest. Dermatol. 102(3): 362-365.

10. Guo, C.-M., R.-L. Wu, S. Feng, and J.-D. Wang. Dracocephalum moldavica L. polysaccharide extraction, determination, and the active oxygen free radical scavenging effect. Food Ferment. Ind. 31(3): 129-132, 2005.

11. Guo X, Cao W, Yao J, Yuan Y, Hong Y, et al. (2015) Cardioprotective effects of tilianin in rat myocardial ischemiareperfusion injury. Mol. Med. Rep. 11(3): 2227-2233. spectrometry, NGM nematode growth medium, PBS phosphatebuffered saline, PVDF polyvinylidene fluoride, ROS reactive oxygen species, SDS sodium dodecyl sulphate, TBS tris buffered saline, UV ultraviolet.

Conflict of interest: F.W., C.P., and T.G. are employees of Mibelle Biochemistry, Mibelle AG. The remaining authors declare that they have no conflict of interest.

12. Hashizume H (2004) Skin Aging, and Dry Skin. J Dermatol. 31(8): 603-609.

13. Ibarra-Alvarado C, Rojas A, Mendoza S, Bah M, Gutiérrez DM, et al. (2010) Vasoactive and antioxidant activities of plants used in Mexican traditional medicine for the treatment of cardiovascular diseaes. Pharm. Biol. 48(7): 732-739.

14. Jiang S, Li T, Yang Z, Yi W, Di S, et al. (2017) AMPK orchestrates an elaborate cascade protecting tissue from fibrosis and aging. Ageing Res. Rev. 38: 18-27.

15. Kenyon CJ (2010) The genetics of aging. Nature 464(7288): 504512.

16. Komatsu T, Park S, Hayashi H, Mori R, Yamaza H, et al. (2019) Mechanisms of Calorie Restriction: A Review of Genes Required for the Life-Extending and Tumor-Inhibiting Effects of Calorie Restriction. Nutrients 11(12): 3068.

17. Maimaitiyiming D, Hu G, Aikemu A, Hu SW, Zhang X (2014) The treatment of Uygur medicine Dracocephalum moldavica L on chronic mountain sickness rat model. Pharmacogn. Mag. 10(40): 477-482.

18. Marrassini C, Cogoi L, Sülsen V, Anesini C (2020) Apigenin-7Glucuronide from Urera aurantiaca Inhibits Tumor Necrosis Factor Alpha and Total Nitrite Release in LipopolysaccharideActivated Macrophages. Evid. Based Complement. Alternat. Med. 2020: 6638764.

19. Martínez-Vázquez M, Estrada-Reyes R, Martínez-Laurrabaquio A, López-Rubalcava C, Heinze G (2012) Neuropharmacological study of Dracocephalum moldavica L. (Lamiaceae) in mice: sedative effect and chemical analysis of an aqueous extract. J. Ethnopharmacol. 141(3): 908-917.

20. Min W, Liu X, Qian Q, Lin B, Wu D, et al. (2014) Effects of Baicalin Against UVA-Induced Photoaging in Skin Fibroblasts. Am. J. Chin. Med. 42(3): 709-727.

21. Mmtiyiming, G. and M. Ayinuer. The clinical curative analysis of Uygur medicine Yixin badiranjibuya granules treats cardiovascular disease. Chin. J. Ethnomed. Ethnopharm. 2:11-14, 2014.

22. Povilaityté V, Venskutonis PR (2000) Antioxidative activity of Purple Peril (Perilla frutescens L.), Moldavian Dragonhead (Dracocephalum moldavica L.), and Roman Chamomile 
(Anthemis nobilis L.) Extracts in Rapeseed Oil. J. Am. Oil Chem. Soc. 77(9): 951-956.

23. Povilaityté V, Cuvelier M-E, Berset C (2001) Antioxidant properties of Moldavian dragonhead (Dracocephalum moldavica L.). J. Food Lipids 8(1): 45-64.

24. Rechinger, H. Labiatae. Flora Iranica. Graz, Austria: Akademische Druck Verlagsanstalt, 1986.

25. Salminen A, Kaarniranta K (2012) AMP-activated protein kinase (AMPK) controls the aging process via an integrated signaling network. Ageing Res. Rev. 11(2): 230-241.

26. Schmid D, Belser E. and Zülli F (2014) Rejuvenating effect of snow algae analyzed. Personal Care Europe 4: 31-34.

27. Schultz MB, Sinclair DA (2016) When stem cells grow old: phenotypes and mechanisms of stem cell aging. Development 143(1): 3-14.

28. Smith Jr. JG, Davidson EA, Sams Jr. WM, Clark RD (1962) Alterations in human dermal connective tissue with age and chronic sun damage. J. Invest. Dermatol. 39: 347-350.

29. Tanaka H, Murakami Y, Ishii I, Nataka S (2009) Involvement of a fork head transcription factor, FOXO1A, in UV-induced changes of collagen metabolism. J. Investig. Dermatol. Symp. Proc. 14(1): 60-62.

30. Teuscher AC, Ewald CY (2018) Overcoming Autofluorescence to Assess GFP Expression During Normal Physiology and Aging in Caenorhabditis elegans. Bio. Protoc. 8(14): e2940.

31. Teuscher AC, Statzer C, Pantasis S, Bordoli MR, Ewald CY (2019) Assessing Collagen Deposition During Aging in Mamallian Tissue and Caenorhabditis elegans. Methods Mol. Biol. 1994: 169-188.

32. Tian Y, Shang J, He T, Cai M, Abdelkader D (2012) Study on material basis of Dracocephalum moldavica for protecting cardiomyocyte against hypoxia/reoxygenation injury by traditional Chinese medicine serum chemical and pharmacological methods. Chinese Journal of Chinese Materia Medica. 37(5): 620-4.

33. Webb AE, Kundaje A, Brunet A (2016) Characterization of the direct targets of FOXO transcription factors throughout evolution. Aging Cell. 15(4): 673-685.

34. Williams DS, Cash A, Hamadani L, Diemer T (2009) Oxaloacetate supplementation increases lifespan in
Caenorhabditis elegans through an AMPK/FOXO-dependent pathway. Aging Cell. 8(6): 765-768.

35. Wu C, Liu H, Rong X, Liu J, Ding W, et al. (2020) Phytochemical composition profile and space-time accumulation of secondary metabolites for Dracocephalum moldavica Linn. via UPLCQ/TOF-MS and HLPC-DAD method. Biomed. Chromatogr. 34(8): e4865.

36. Xing J, Peng K, Cao W, Lian X, Wang Q, et al. (2013) Effects of total flavonoids from Dracocephalum moldavica on the proliferation, migration, and adhesion molecule expression of rat vascular smooth muscle cells induced by TNF- $\alpha$. Pharm. 1 Biol. 51(1): 74-83.

37. Yang L-N, Xing J-G, He C-H, Wu T (2014) The phenolic compounds from Dracocephalum moldavica L. Biochem. Syst. Ecol. 54: 19-22.

38. Yang L-N, Xing J-G, He C-H, Yang P-M (2013) Chemical constituents of Dracocephalum Moldavica L. and their pharmacological activities. World Clin. World Clin. Drugs 34(4): 226-31.

39. Yu N, He CH, Awuti G, Zeng C, Xing JG et al. (2015) Simultaneous Determination of Six Active Compounds in Yixin Badiranjibuya Granules, a Traditional Chinese Medicine, by RPHPLC-UV Method. J. Anal. Methods Chem. 2015: 974039.

40. Yun H, Park S, Kim M-J, Yang WK, Im DU, et al. (2014) AMPactivated protein kinase mediates the antioxidant effects of resveratrol through regulation of the transcription factor FoxO1. FEBS J. 281(19): 4421-4438.

41. Zhang S, Duan E (2018) Fighting against Skin Aging: The Way from Bench to Bedside. Cell Transplant. 27(5): 729-738.

42. Zheng R-F, Du Y-W, Zeng C, Wang H-F, Xing J-G, et al. (2020) Total flavones of Dracocephalum moldavica L. protect astrocytes against $\mathrm{H}_{2} \mathrm{O}_{2}$-induced apoptosis through a mitochondriadependent pathway. BMC Complement. Med. and Ther. 20(1): 78.

43. Zhou B-R, Luo D, Wei F-D, Chen X-E, Gao J (2008) Baicalin protects human fibroblasts against ultraviolet B-induced cyclobutane pyrimidine dimers formation. Arch. Dermatol. Res. 300(6): 331-4. 